\title{
Reduced Order Modeling for Multi-scale Control of Low Temperature Combustion Engines
}

\author{
Eugen Nuss, Dennis Ritter, Maximilian Wick, Jakob Andert, \\ Dirk Abel and Thivaharan Albin
}

\begin{abstract}
Internal combustion engines face tightening limits on pollutant and greenhouse gas emissions. Therefore, new solutions for clean combustion have to be found. Low Temperature Combustion is a promising technology in this regard, as it is able to reduce pollutant emissions while increasing the engine's efficiency. Recent research has shown that closed-loop control manages to stabilize the process. Nevertheless, sensitivity to varying boundary conditions and a narrow operating range remain unfavorable. To investigate new control concepts such as in-cycle feedback, computationally feasible cycle-resolved models become necessary. This work presents a low order model for Gasoline Controlled Auto Ignition (GCAI) that is continuous in time and computes the pressure trace over the entire combustion cycle. A comparison between simulation and measurement supports the suitability of the modeling approach. Furthermore, the model captures the characteristic transition of system dynamics in case GCAI during late combustion.
\end{abstract}

\footnotetext{
E. Nuss · D. Ritter · D. Abel

Institute of Automatic Control, RWTH Aachen University,

Campus-Boulevard 30, 52074 Aachen, Germany

e-mail: e.nuss@irt.rwth-aachen.de

D. Ritter

e-mail: d.ritter@irt.rwth-aachen.de

D. Abel

e-mail: d.abel@irt.rwth-aachen.de
}

M. Wick · J. Andert

Institute for Combustion Engines, RWTH Aachen University,

Forckenbeckstraße 4, 52074 Aachen, Germany

e-mail: Wick_m@vka.rwth-aachen.de

\section{J. Andert}

e-mail: andert@mechatronics.rwth-aachen.de

T. Albin (凶)

Institute for Dynamic Systems and Control, ETH Zürich, Sonneggstrasse 3,

8092 Zürich, Switzerland

e-mail: albinrat@ethz.ch 
Keywords Gasoline controlled autoignition - Low temperature combustion Low order HCCI modeling

\section{Introduction}

Combustion engines are a crucial mobile drive for transportation purposes due to the characteristics of good availability, high energy density and easy storage capability of liquid fuels. An important goal for the future is the reduction of combustion related pollutant emissions and greenhouse gases.

A promising technology to improve the combustion process is Low Temperature Combustion (LTC). LTC promises to fulfill existing requirements on power and high efficiency while simultaneously reducing pollutant emissions. Additionally, it is applicable to both diesel and gasoline engines. The latter is called Gasoline Controlled Auto Ignition (GCAI) in this paper. A common property of different LTC applications is the high degree of homogenization of the air-fuel mixture and the need of self-ignition to trigger the combustion.

While the LTC concept has significant advantages, challenges arise with respect to process stabilization. The process of LTC is substantially determined by chemical reaction kinetics which leads to a lack of the stabilizing mechanism of the mixturecontrolled high-temperature combustion. As result, LTC has a high sensitivity with respect to the global and local thermodynamic state in the combustion chamber. The thermodynamic state is characterized by a high number of parameters such as temperature, chemical composition and stratification. To reduce the sensitivity to these parameters, the application of closed-loop control methods has been established.

Very roughly speaking, the general stabilization of the process has been shown in different research groups. However, there are still two aspects which cause the necessity for further development in this field. On one side, the limited operating range remains unfavourable. On the other side, stabilization of the process under all possible boundary conditions still needs to be ensured.

Conventional control techniques operate on a cycle-to-cycle basis. For each combustion cycle, a surrogate parameter is calculated which represents the combustion behaviour. This parameter is used as a controlled variable within the closed-loop controller. Typical values are the indicated mean effective pressure (IMEP), the crank-angle of $50 \%$ burned fuel mass (CA50) or the maximum pressure rise gradient (DPMAX). The cycle basis also results in updating the actuated values only once per cycle. In this way, disturbances which act on the current combustion cycle cannot be rejected.

A possibility to consider the disturbances which arise on a smaller time scale are control strategies which can be classified as multi-scale control algorithms. These algorithms allow for in-cycle cylinder control where the feedback is based on the measurements of the current cycle. First experiments to show the feasibility of this approach can be found in [1]. An important factor for the successful application are new reduced order models for the purpose of control design. In contrast to mean 
Fig. 1 Pressure trace of GCAI with exhaust gas recompression-different sections of one cycle and the Negative Valve Overlap (NVO)

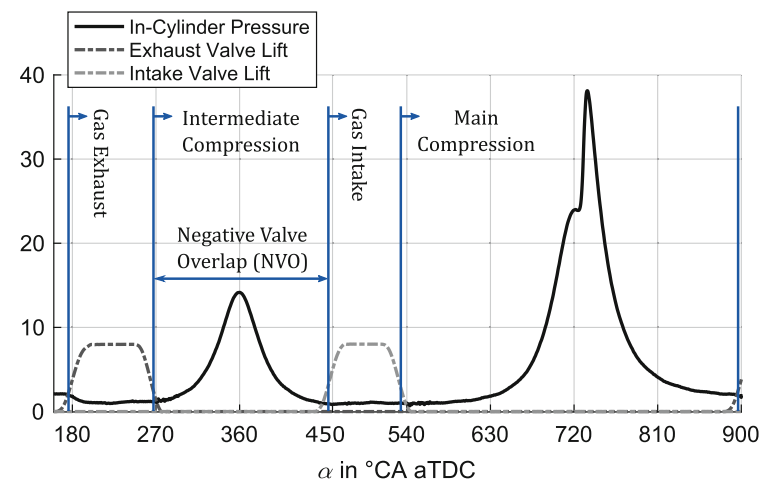

value models, these need to capture the dynamics of the entire cylinder pressure and also have low order to be suitable for control design.

In this paper, an innovative modeling approach for the purpose of multi-scale control for GCAI engines is investigated. The main requirement lies in a model with low complexity that is capable of capturing the entire cylinder pressure trace. The particular process studied uses a fully variable valve train in combination with exhaust gas recompression for process control. The flexible choice of valve timings enables a symmetric collocation of exhaust valve closing and inlet valve opening around the gas exchange Top Death Center (TDC) (see Fig. 1). The resulting Negative Valve Overlap (NVO) retains an amount of the residual gas inside the cylinder and thus influences the dilution and temperature of the charge. This in turn affects the auto ignition, which is used to control the combustion timing [2].

Section 2 presents the control-oriented, time-continous GCAI model for the specified GCAI process. In Sect. 3, simulation results are compared to experimental data of a GCAI test bed. Finally, Sect. 4 concludes the paper with the summary and drawn conclusions.

\section{Gasoline Controlled Auto-Ignition Model}

Recent research has shown that a cycle-to-cycle control approach generally enables GCAI operation [3, 4]. Multi-scale control methods hold promise for further improvement in terms of process stabilization and extension of the operating range. But these methods require control oriented system models that reproduce the in-cycle behavior. Despite the underlying nonlinear process characteristics, the model's complexity must be limited to be suitable for control synthesis. This section introduces a first principle based zero-dimensional modeling approach for GCAI.

Events that only last few degrees of crank-angle (CA) relative to the entire engine cycle have time constants that are small compared to other processes within the engine cycle. Therefore, the exhaust valve opening (EVO), exhaust valve closing (EVC), 
(a)

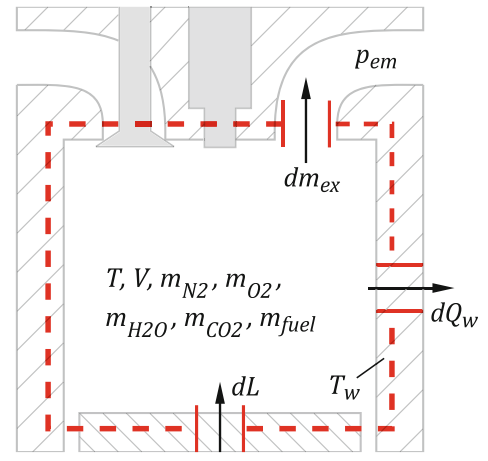

(c)

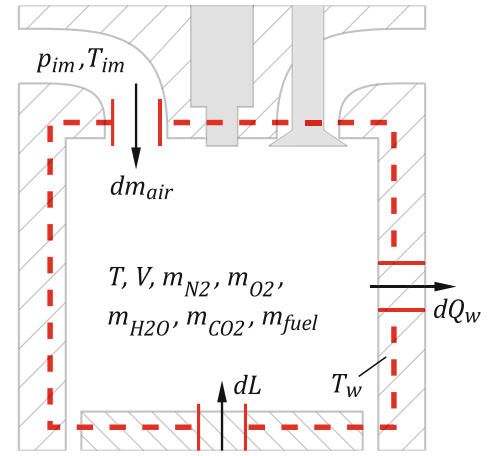

(b)

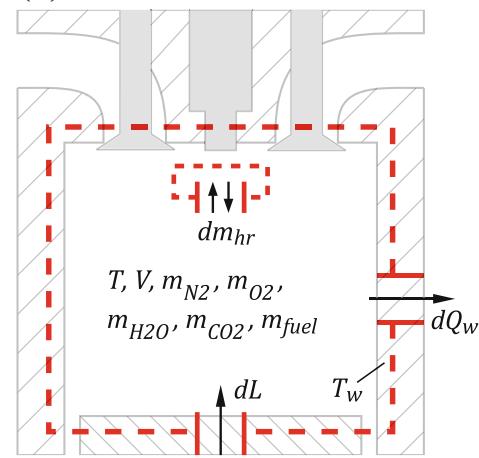

(d)

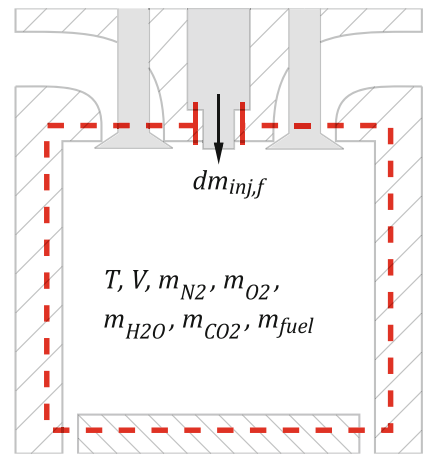

Fig. 2 GCAI stages. a Gas exhaust (ex). b Intermediate or main compression (ic/mc). c Gas intake (in). d Fuel injection (inj, f)

inlet valve opening (IVO), inlet valve closing (IVC) as well as the fuel injection (inj, f) are modeled as zero-time events. In case of the valve events, opening or closing is assumed when $10 \%$ of the maximum valve lift is reached. The resulting discrete valve timings divide the engine cycle into the four seperate stages gas exhaust (ex), intermediate compression (ic), gas intake (in) and main compression (mc). Figure 2a-c show the distinct system dynamics of each stage according to the current valve positions. The fuel's heat release can take place during both, intermediate and main compression. Despite being included in schematic (b), the combustion event is also modeled as a discrete time event and addressed specifically later in this section. Contrary to the valve timings, the fuel injection does not separate two different system dynamics, but causes a discontinuous jump in the system states. The underlying physically motivated equations of this impulsive event are based on Fig. 2d. 
The resulting multi-stage model with eight states and six control inputs is:

$$
\begin{aligned}
\frac{d x}{d t} & =f_{i}(x) \quad i=e x, i c, i n, m c \\
x\left(t_{0, i}\right) & =x\left(t_{\text {end }, i-1}\right) \\
y & =h(x) \\
x & =\left(T, \alpha, m_{N_{2}}, m_{O_{2}}, m_{H_{2} O}, m_{C O_{2}}, m_{f u e l}, T_{w}\right)^{T} \\
u & =\left(\alpha_{e v o}, \alpha_{e v c}, \alpha_{i v o}, \alpha_{i v c}, \alpha_{s o i}, m_{i n j, f}\right)^{T} \\
y & =p
\end{aligned}
$$

where $x$ is the state, $u$ the input and $y$ the output vector.

The engine model repetitively develops through the continuous state spaces $f_{i}(x)$ of each introduced stage in the defined order. The valve timings actuate as external switches between the stages. When an external switch is triggered, the state of the current stage serves as the initial condition for following one.

According to Fig.2, the state vector $x$ of all functions $f_{i}(x)$ consists of the in-cylinder temperature $T$, the crank angle $\alpha$, the masses of the five species $m_{N_{2}}, m_{\mathrm{O}_{2}}, m_{\mathrm{H}_{2} \mathrm{O}}, m_{\mathrm{CO}_{2}}$ and $m_{f u e l}$ as well as the temperature $T_{w}$ of the walls surrounding the charge. Throughout the work, iso-octane $\mathrm{C}_{8} \mathrm{H}_{18}$ is assumed as fuel. The valve timings are expressed through the crank-angle at which the valve opening or closing takes place, stated in ${ }^{\circ} \mathrm{CA}$ after Top Death Center (aTDC). Further control inputs are the crank-angle at start of fuel injection $\alpha_{s o i}$ and the injected fuel mass $m_{i n j, f}$. Due to modeling the valve timings as external switches and the fuel injection as a discrete jump, none of the control inputs directly appears in $f_{i}(x)$. The only model output is the in-cylinder pressure $p$. The following subsections present the physical and phenomenological equations the model is based on.

\subsection{Energy Balance}

The general energy balance covering all cases of Fig. 2 is used in the form:

$$
\mathrm{d} U=\mathrm{d} Q_{w}+\mathrm{d} L+h_{\text {in }} \cdot \mathrm{d} m_{\text {in }}+h \cdot \mathrm{d} m_{\text {out }}
$$

First, Eq. 2 is further specified by considering only compression work and assuming ideal gases. Second, the terms including the differential of specific internal energy $\mathrm{d} u$ and species $\mathrm{d} Y_{k}$ are combined in order to achieve a direct dependence on the species' masses and eliminate the differential of the internal energy.

$$
\mathrm{d} U=\mathrm{d} Q_{w}-\frac{m \cdot R \cdot T}{V} \mathrm{~d} V+h_{\text {in }} \cdot \mathrm{d} m_{\text {in }}+h \cdot \mathrm{d} m_{\text {out }}
$$




$$
\begin{aligned}
\mathrm{d} U & =m \cdot \mathrm{d} u+m \cdot c_{v} \cdot \mathrm{d} T+\sum_{k} u_{k} \cdot \mathrm{d} Y_{k} \\
& =m \frac{R}{\kappa-1} \mathrm{~d} T+\sum_{k} u_{k} \cdot \mathrm{d} m_{k}
\end{aligned}
$$

Solving for $\mathrm{d} T$ and deriving with respect to time yields the general differential equation for the in-cylinder temperature:

$$
\Rightarrow \dot{T}=\frac{\kappa-1}{m \cdot R}\left(\dot{Q}_{w}-\frac{m \cdot R \cdot T}{V} \cdot \dot{V}+h_{\text {in }} \cdot \dot{m}_{\text {in }}+h \cdot \dot{m}_{\text {out }}-\sum_{k} u_{k} \cdot \dot{m}_{k}\right)
$$

Applying the simplifications for the stages according to Fig. 2 results in:

$$
\begin{aligned}
& \dot{T}_{e x}=\frac{\kappa-1}{m \cdot R}\left(\dot{Q}_{w}-\frac{m \cdot R \cdot T}{V} \cdot \dot{V}+h \cdot \dot{m}_{e x}-\sum_{k} u_{k} \cdot \dot{m}_{k}\right) \\
& \dot{T}_{i c}=\dot{T}_{m c}=\frac{\kappa-1}{m \cdot R}\left(\dot{Q}_{w}-\frac{m \cdot R \cdot T}{V} \cdot \dot{V}\right) \\
& \dot{T}_{i n}=\frac{\kappa-1}{m \cdot R}\left(\dot{Q}_{w}-\frac{m \cdot R \cdot T}{V} \cdot \dot{V}+h_{\text {air }} \cdot \dot{m}_{\text {air }}-\sum_{k} u_{k} \cdot \dot{m}_{k}\right)
\end{aligned}
$$

As the combustion event is treated seperately, Eq. 6 reduces to Eq. 8 for the intermediate and main compression.

\subsection{Mass Balance}

The change in the total charge mass during the exhaust and intake stroke, respectively, is evaluated by the valve flow equations [5]:

$$
\begin{aligned}
& \dot{m}_{e x}=A_{s, e x} \cdot \sqrt{\frac{2 \cdot p}{R \cdot T}} \cdot \sqrt{\frac{\kappa}{\kappa-1}\left(\left(\frac{p_{e m}}{p}\right)^{\frac{2}{\kappa}}-\left(\frac{p_{\text {em }}}{p}\right)^{\frac{\kappa+1}{\kappa}}\right)} \\
& \dot{m}_{\text {in }}=A_{s, \text { in }} \cdot \sqrt{\frac{2 \cdot p_{\text {im }}}{R_{\text {air }} \cdot T_{\text {im }}}} \cdot \sqrt{\frac{\kappa_{\text {air }}}{\kappa_{\text {air }}-1} \cdot\left(\left(\frac{p}{p_{\text {im }}}\right)^{\frac{2}{\kappa_{\text {air }}}}-\left(\frac{p}{p_{\text {im }}}\right)^{\frac{\kappa_{\text {air }}+1}{\kappa_{\text {air }}}}\right)}
\end{aligned}
$$

where $A_{s}$ represents the valve specific isentropic flow cross-sectional area and the in-cylinder pressure $p$ is determined through the ideal gas law. Depending on the stage, either fresh air is inducted into the cylinder or the in-cylinder gases flow into the exhaust manifold. 


\subsection{Species Balance}

During the exhaust stroke, the mass fractions remain constant as the charge is assumed to be a homogeneous mixture. Using Eq. 10, the following relation holds for all species during the exhaust stroke:

$$
\dot{m}_{k, e x}=Y_{k} \cdot \dot{m}_{e x}, \quad k=N_{2}, \mathrm{O}_{2}, \mathrm{H}_{2} \mathrm{O}, \mathrm{CO}_{2}, \text { fuel }
$$

During the gas intake phase, only air with a constant mass distribution is inducted. Consequently, only the masses of $\mathrm{N}_{2}$ and $\mathrm{O}_{2}$ change according to:

$$
\begin{aligned}
& \dot{m}_{N 2, \text { in }}=Y_{N 2, \text { air }} \cdot \dot{m}_{\text {air }} \\
& \dot{m}_{O 2, \text { in }}=Y_{O 2, \text { air }} \cdot \dot{m}_{\text {air }}
\end{aligned}
$$

\subsection{Heat Release and Fuel Injection}

Heat release during main and intermediate combustion as well as the fuel injection are modeled as discrete jumps in the state vector $x$. For these zero-time events, the piston position does not change and wall heat losses are neglected. Starting from Eq. 6, the discrete step in the in-cylinder temperature due to heat release is:

$$
\mathrm{d} T_{h r}=\frac{1-\kappa}{m \cdot R} \cdot \sum_{k} u_{k} \cdot \mathrm{d} m_{k}
$$

The global combustion reaction for iso-octane establishes a relation between amount of burned fuel $m_{f, h r}$ and the modeled species. In order to match the models units, the reaction equation is transformed with the respective mole masses.

$$
\begin{aligned}
\mathrm{C}_{8} \mathrm{H}_{18}+\mathrm{O}_{2} & \rightarrow \mathrm{CO}_{2}+\mathrm{H}_{2} \mathrm{O} \\
\Rightarrow c_{f u e l} \cdot\left[\mathrm{C}_{8} \mathrm{H}_{18}\right]_{k g}+c_{\mathrm{O} 2} \cdot\left[\mathrm{O}_{2}\right]_{k g} & \rightarrow c_{\mathrm{CO} 2} \cdot\left[\mathrm{CO}_{2}\right]_{k g}+c_{\mathrm{H} 2 \mathrm{O}} \cdot\left[\mathrm{H}_{2} \mathrm{O}\right]_{k g}
\end{aligned}
$$

The introduced constants are listed in Table 1. The change in the species can now be stated in dependence of the burned fuel mass:

$$
\mathrm{d} m_{k}=c_{k} \cdot m_{f, h r}, \quad k=\mathrm{O}_{2}, \mathrm{H}_{2} \mathrm{O}, \mathrm{CO}_{2}, \text { fuel }
$$

A difference between intermediate and main combustion lies in determining the amount of burned fuel. As for the intermediate compression, the amount of burned fuel is assumed proportional to the remaining fuel in the cylinder. The introduced 
Table 1 Model parameters

\begin{tabular}{l|l|l|l|l|l|l|l}
\hline Param. & Value & Unit & Eq & Param. & Value & Unit & Eq \\
\hline$h_{\text {air }}$ & $2.5313 \mathrm{e}+04$ & $\mathrm{~J}_{\mathrm{kg}^{-1}}$ & 9 & $\beta_{3}$ & $-2.5139 \mathrm{e}+02$ & - & 19 \\
\hline$A_{s, \text { ex }}$ & $1.5000 \mathrm{e}-04$ & $\mathrm{~m}^{2}$ & 10 & $\beta_{4}$ & $4.0000 \mathrm{e}-01$ & - & 20 \\
\hline$p_{\text {em }}$ & $1.0000 \mathrm{e}+05$ & $\mathrm{~Pa}$ & 10 & $\gamma_{0}$ & $5.4790 \mathrm{e}-01$ & $\mathrm{rad}$ & 21 \\
\hline$A_{s, \text { in }}$ & $2.0000 \mathrm{e}-04$ & $\mathrm{~m}^{2}$ & 11 & $\gamma_{1}$ & $1.9696 \mathrm{e}+05$ & $\mathrm{~Pa}^{-\gamma 2}$ & 21 \\
\hline$p_{\text {im }}$ & $1.0000 \mathrm{e}+05$ & $\mathrm{~Pa}$ & 11 & $\gamma_{2}$ & $7.0670 \mathrm{e}-01$ & - & 21 \\
\hline$T_{\text {im }}$ & $3.2315 \mathrm{e}+02$ & $\mathrm{~K}$ & 11 & $\gamma_{3}$ & $6.5131 \mathrm{e}+03$ & $\mathrm{~K}$ & 21 \\
\hline$\kappa_{\text {air }}$ & $1.3980 \mathrm{e}+00$ & - & 11 & $\gamma_{4}$ & $1.3270 \mathrm{e}+00$ & - & 22 \\
\hline$R_{\text {air }}$ & $2.8818 \mathrm{e}+02$ & $\mathrm{~J} . \mathrm{kg}^{-1} K^{-1}$ & 11 & $\gamma_{5}$ & $-4.0457 \mathrm{e}+00$ & $\mathrm{rad}$ & 22 \\
\hline$Y_{N_{2}, \text { air }}$ & $7.6700 \mathrm{e}-01$ & - & 13 & $h_{\text {evap }, f}$ & $2.6954 \mathrm{e}+05$ & $\mathrm{~J} \mathrm{~kg}$ & 23 \\
\hline$Y_{O_{2}, \text { air }}$ & $2.3300 \mathrm{e}-01$ & - & 14 & $h_{\text {inj, },}$ & $-1.9142 \mathrm{e}+06$ & $\mathrm{~J} . \mathrm{kg}^{-1}$ & 23 \\
\hline$c_{\text {fuel }}$ & $-1.0000 \mathrm{e}+00$ & - & 17 & $D$ & $8.4000 \mathrm{e}-02$ & $\mathrm{~m}$ & 24 \\
\hline$c_{O 2}$ & $-3.5088 \mathrm{e}+00$ & - & 17 & $T_{w 1}$ & $1.2000 \mathrm{e}-01$ & $\mathrm{~s}$ & 25 \\
\hline$c_{C O 2}$ & $3.0877 \mathrm{e}+00$ & - & 17 & $\delta_{0}$ & $3.5000 \mathrm{e}+02$ & $\mathrm{~K}$ & 25 \\
\hline$c_{H 2 O}$ & $1.4211 \mathrm{e}+00$ & - & 17 & $V_{c}$ & $5.2501 \mathrm{e}-05$ & $\mathrm{~m}^{3}$ & 32 \\
\hline$\beta_{0}$ & $9.5000 \mathrm{e}-01$ & - & 19 & $r_{1}$ & $4.5000 \mathrm{e}-02$ & $\mathrm{~m}$ & 32 \\
\hline$\beta_{1}$ & $1.9757 \mathrm{e}+01$ & $\mathrm{rad}^{-1}$ & 19 & $r_{2}$ & $1.5900 \mathrm{e}-01$ & $\mathrm{~m}$ & 32 \\
\hline$\beta_{2}$ & $-3.9793 \mathrm{e}+05$ & $\mathrm{~kg}^{-1}$ & 19 & $f u e l$ & $C_{8} H_{18}$ & - & - \\
\hline
\end{tabular}

proportional constant $\beta_{4}$ is eventually tuned to fit the model to measurement data. For the main compression on the other hand, a varying combustion efficiency $\eta_{h r}$ is introduced, which is initially presented in [6]. These incomplete combustions lead to a dynamic coupling between consecutive cycles.

$$
\begin{aligned}
m_{f, h r, m c} & =\beta_{0} \cdot\left(1+e^{\beta_{1} \cdot C A 50+\beta_{2} \cdot m_{i n j, f}+\beta_{3}}\right)^{-1} \cdot m_{f u e l} \\
m_{f, h r, i c} & =\beta_{4} \cdot m_{f u e l}
\end{aligned}
$$

Furthermore, it is necessary to determine the instant at which the combustion occurs. During main compression, the center of combustion CA50 is used and identified by applying a semi-empirical Arrhenius rate threshold approach [7]. The integral

$$
\gamma_{0}=\int_{\alpha_{i v c}}^{\alpha_{s o c}} \gamma_{1} \cdot p^{\gamma_{2}} \cdot e^{-\frac{\gamma_{3}}{T}} d \alpha
$$

is numerically integrated alongside the state space model until it reaches the prescribed threshold $\gamma_{0}$. The crank-angle at this instance represents the start of combustion. Linear extrapolation yields

$$
C A 50=\gamma_{4} \cdot \alpha_{s o c}+\gamma_{5}
$$


Analogous to [6], the heat release during the intermediate compression is modeled in the beginning of the stage.

The only change in the species' masses caused by fuel injection trivially is $\mathrm{d} m_{\text {fuel }}=$ $m_{i n j, f}$. The corresponding change in the in-cylinder temperature is:

$$
\mathrm{d} T_{i n j, f}=\frac{\kappa-1}{m \cdot R}\left(h_{i n j, f}-h_{e v a p, f}-u_{i n j, f}\right) \cdot m_{i n j, f}
$$

\subsection{Wall Heat Loss}

The wall heat losses $\dot{Q}_{w}$ are calculated by using the Hohenberg correlation [8] for estimating the heat transfer coefficient $\alpha_{w}$ and the ideal gas law for the in-cylinder pressure $p$.

$$
\begin{aligned}
\dot{Q}_{\text {wall }} & =\alpha_{w} \cdot A_{w} \cdot\left(T_{w}-T\right) \\
\alpha_{w} & =130 \cdot V^{-0.06} \cdot\left(\frac{p}{10^{5}}\right)^{0.8} \cdot T^{-0.4} \cdot\left(\frac{4 \cdot \dot{V}}{\pi \cdot D^{2}}+1.4\right)^{0.8} \\
A_{w} & =\frac{4 \cdot V}{D}+\frac{\pi \cdot D^{2}}{2}
\end{aligned}
$$

The dynamics of the wall temperature $T_{w}$ are approximated by a first order lag element with the in-cylinder temperature $T$ as the input:

$$
\dot{T}_{w}=\frac{1}{T_{w, 1}}\left(T-T_{w}-\delta_{0}\right)
$$

The time constant $T_{w, 1}$ is initially chosen so that a step response in reaches a stationary level after three cycles. Additionally a constant cooling factor $\delta_{0}$ is introduced and determined by tuning the paramater to fit measurement data.

\subsection{Auxilary Equations}

The derived set of equations still depends on a set of parameters that can partly be expressed through functional relations. The component's specific heat capacity $c_{p, k}$ and enthalpy $h_{k}$ are expressed through the in-cylinder temperature $T$ by using NASA polynomials, as: 


$$
\begin{aligned}
c_{p, k} & =\left(a_{1}+a_{2} \cdot T+a_{3} \cdot T^{2}+a_{4} \cdot T^{3}+a_{5} \cdot T^{4}\right) \frac{\bar{R}}{\bar{M}_{k}} \\
h_{k} & =\left(a_{1} \cdot T+a_{2} \cdot T^{2}+a_{3} \cdot T^{3}+a_{4} \cdot T^{4}+a_{5} \cdot T^{5}+a_{6}\right) \frac{\bar{R}}{\bar{M}_{k}}
\end{aligned}
$$

Given $c_{p, k}$ and $h_{k}$, the specific enthalpy $h$, the isentropic exponent $\kappa$, the specific gas constant $R$ and the internal energy $u_{k}$ of each component $k$ are determined with:

$$
\begin{aligned}
h & =\sum_{k} Y_{k} \cdot h_{k} \\
c_{p} & =\sum_{k} Y_{k} \cdot c_{p, k} \\
R & =\sum_{k} Y_{k} \cdot \frac{\bar{R}}{\overline{M_{k}}} \\
\kappa & =\frac{c_{p}}{c_{p}-R} \\
u_{k} & =h_{k}-\frac{\bar{R}}{\bar{M}_{k}} T
\end{aligned}
$$

As the state vector contains the mass of each modeled component, $Y_{k}$ can always be directly computed.

The current cylinder volume $V$ and its time derivative $\dot{V}$ are gained through the slider-crank formula [5] with the bore diameter $D$, the volume at top death center $V_{c}$ and the two rod lengths $r_{1}$ and $r_{2}\left(\lambda=\frac{r_{2}}{r_{1}}\right)$ :

$$
\begin{aligned}
& V=V_{c}+\frac{\pi \cdot D^{2}}{4}\left(r_{1} \cdot\left(1-\sqrt{1-\lambda^{2} \cdot \sin (\alpha)^{2}}\right)+r_{2} \cdot(1-\cos (\alpha))\right) \\
& \dot{V}=\frac{\pi \cdot D^{2}}{4}\left(r_{1} \cdot \frac{\lambda^{2} \cdot \cos (\alpha)}{\sqrt{1-\lambda^{2} \cdot \sin (a)^{2}}}+r_{2}\right) \cdot \omega \cdot \sin (\alpha)
\end{aligned}
$$

\subsection{Model Summary}

The complete set of state space functions is as follows:

$$
\begin{aligned}
f_{e x}(x)= & \left(\dot{T}_{e x}, \omega, \dot{m}_{e x} \cdot Y_{N_{2}}, \dot{m}_{e x} \cdot Y_{O_{2}}, \dot{m}_{e x} \cdot Y_{H_{2} O}, \ldots\right. \\
& \left.\dot{m}_{e x} \cdot Y_{C O_{2}}, \frac{1}{T_{w, 1}}\left(T-T_{w}-\delta_{0}\right)\right)^{T} \\
f_{i c}(x)= & \left(\dot{T}_{i c}, \omega, 0,0,0,0,0, \frac{1}{T_{w, 1}}\left(T-T_{w}-\delta_{0}\right)\right)^{T}
\end{aligned}
$$




$$
\begin{gathered}
f_{i n}(x)=\left(\dot{T}_{i n}, \omega, \dot{m}_{i n} \cdot Y_{N_{2}, a i r}, \dot{m}_{i n} \cdot Y_{O_{2}, a i r}, \ldots\right. \\
\left.0, \quad 0, \quad 0, \frac{1}{T_{w, 1}}\left(T-T_{w}-\delta_{0}\right)^{T}\right) \\
f_{m c}(x)=\left(\dot{T}_{m c}, \omega, 0,0,0,0,0, \frac{1}{T_{w, 1}}\left(T-T_{w}-\delta_{0}\right)\right)^{T}
\end{gathered}
$$

The engine speed $n$ is substituted by $\omega$ in order to emphasize the use of SI-units in the model. The discrete state jumps due to fuel injection and combustion are:

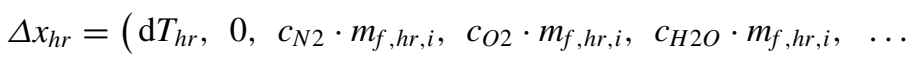

$$
\begin{aligned}
& \left.c_{C O 2} \cdot m_{f, h r, i}, \quad c_{C 8 H 18} \cdot m_{f, h r, i}, 0\right)^{T} \quad i=i c, m c
\end{aligned}
$$

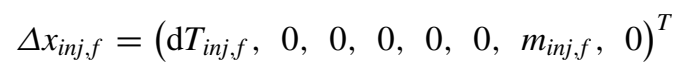

Figure 3 illustrates the implementation of the hybrid multi-stage model. Once the crank-angle $\alpha$ crosses a control input in form of a valve opening or closing, the subsequent state space function becomes active and uses the current state vector as an initial condition. The conversion of remaining fuel during intermediate compression is modeled through a discrete combustion event in the beginning of the phase. With regard to implementation, this results in a manipulation of the respective initial condition. Fuel injection also takes place during the intermediate compression and adds an offset to the state vector at the start of injection $\alpha_{\text {soi }}$. The integrator is reset with the updated state during this step. The main combustion is triggered through the Arrhenius ignition delay model and produces a step in the state vector at the center of combustion $C A 50$. In contrast to the intermediate combustion the amount of burned fuel is not constant but varies according to the empirical combustion efficiency. All parameters of the model are listed in Table 1.

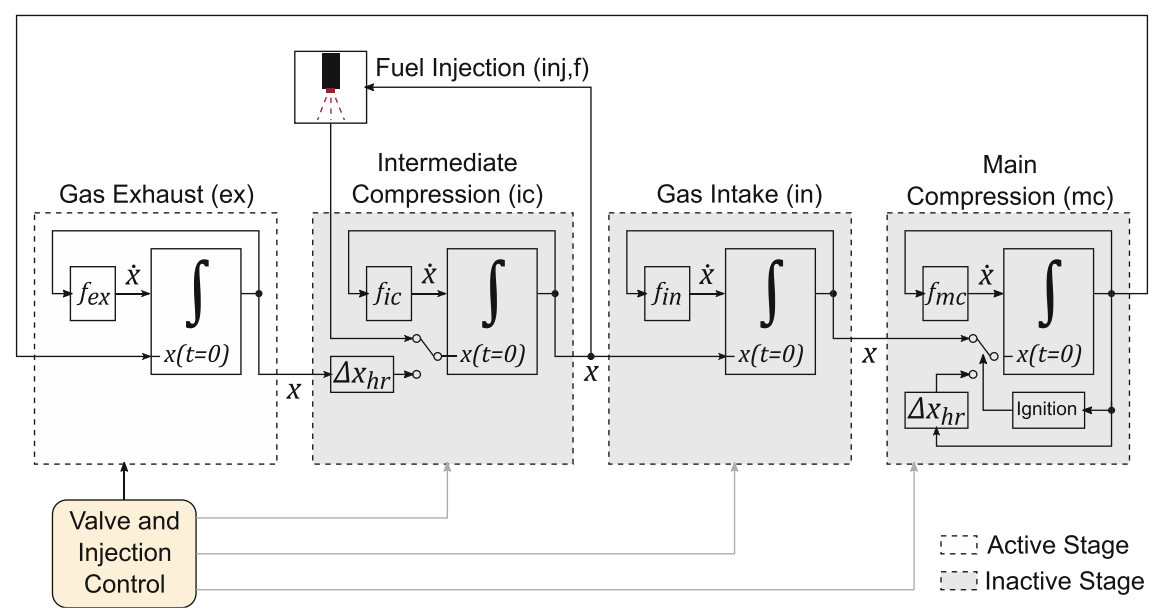

Fig. 3 Schematic of the continuous-cycle GCAI model—shown during the exhaust stroke 


\section{Simulation Results}

In this section, the simulated output of the model presented in Sect. 2 is compared to measurement data. In the experimental setup, 14 different NVO settings are recorded at an engine speed of $1500 \mathrm{rpm}$. Each of the 14 measurement points contains 200 consecutive cycles, excluding the transition from one measurement point to the other. Regarding the system inputs, EVC and IVO are set symmetrically to the gas exchange TDC and are adapted to achieve an NVO sweep from $190^{\circ}-183^{\circ} \mathrm{CA}$. EVO, IVC and the injected fuel mass remain constant. For reasons of better homogenization, the start of fuel injection $\alpha_{\text {soi }}$ is referenced to the intake valve opening event.

The discrete valve timings necessary for simulation are extracted from the valve lifts of each measured cycle. Also in the simulation, each measurement point is held for 200 cycles. The simulation step size is set to achieve a crank-angle discretization $\Delta \alpha_{\text {sim }}$ of $0.1^{\circ} \mathrm{CA}$. Table 2 lists the set points for the valve timings and the remaining parameters useds for simulation.

Figure 4 compares the simulated and the measured pressure trace of an arbitrary cycle for three different measurement points. As the experimental raw data is recorded with a continuous advancement in the crank-angle, the simulation results are adapted and plotted over steadily increasing ${ }^{\circ} \mathrm{CA}$ instead of ${ }^{\circ} \mathrm{CA}$ a TDC which is reset each $720^{\circ}$.

In general, the model achieves good resemblance with regard to the measured pressure trace. The intermediate compression is fit well in all three cases. Considering the main compression, the decrease starting from the point of maximum pressure is approximated well also. The maximum pressure peak, however, is overestimated in case (a), while the simulated CA50 is inaccurate in all three cases, varying up to $5.5^{\circ} \mathrm{CA}$.

As it is unpractical to compare the large number of cycles in detail, the center of combustion CA50 of all measured and simulated cycles is shown in the return map in Fig. 5a. In case of the model, CA50 can directly be extracted while the measurement data was processed by heat release analysis. With reducing NVO, also the temperature of the cylinder charge decreases and consequently the combustion center CA50 shifts towards later combustion. Again, the overall fit, particularly the covered range of CA50 of approximately $4-12^{\circ} \mathrm{CA} a \mathrm{TDC}$, is acceptable. However, the measured data include some non gaussian outliers the model does not capture. Reaching late combustion in terms of CA50, GCAI enters a specific behaviour that has been intensively investigated $[1,9,10]$. Figure $5 \mathrm{~b}$ shows a qualitative comparison

Table 2 Simulation parameters

\begin{tabular}{l|l|l|l|l|l}
\hline Param. & Value & Unit & Param. & Value & Unit \\
\hline$\alpha_{\text {evo }}$ & 160 & ${ }^{\circ} \mathrm{CA} a$ TDC & NVO & $190-183$ & ${ }^{\circ} \mathrm{CA}$ \\
\hline$\alpha_{\text {ivc }}$ & 545 & ${ }^{\circ} \mathrm{CA} a$ TDC & $\Delta \alpha_{\text {sim }}$ & 0.1 & ${ }^{\circ} \mathrm{CA}$ \\
\hline$\alpha_{\text {soi }}$ & $455-448.5$ & ${ }^{\circ} \mathrm{CA} a \mathrm{TDC}$ & $n_{\text {engine }}$ & 15000 & $\mathrm{rpm}$ \\
\hline
\end{tabular}



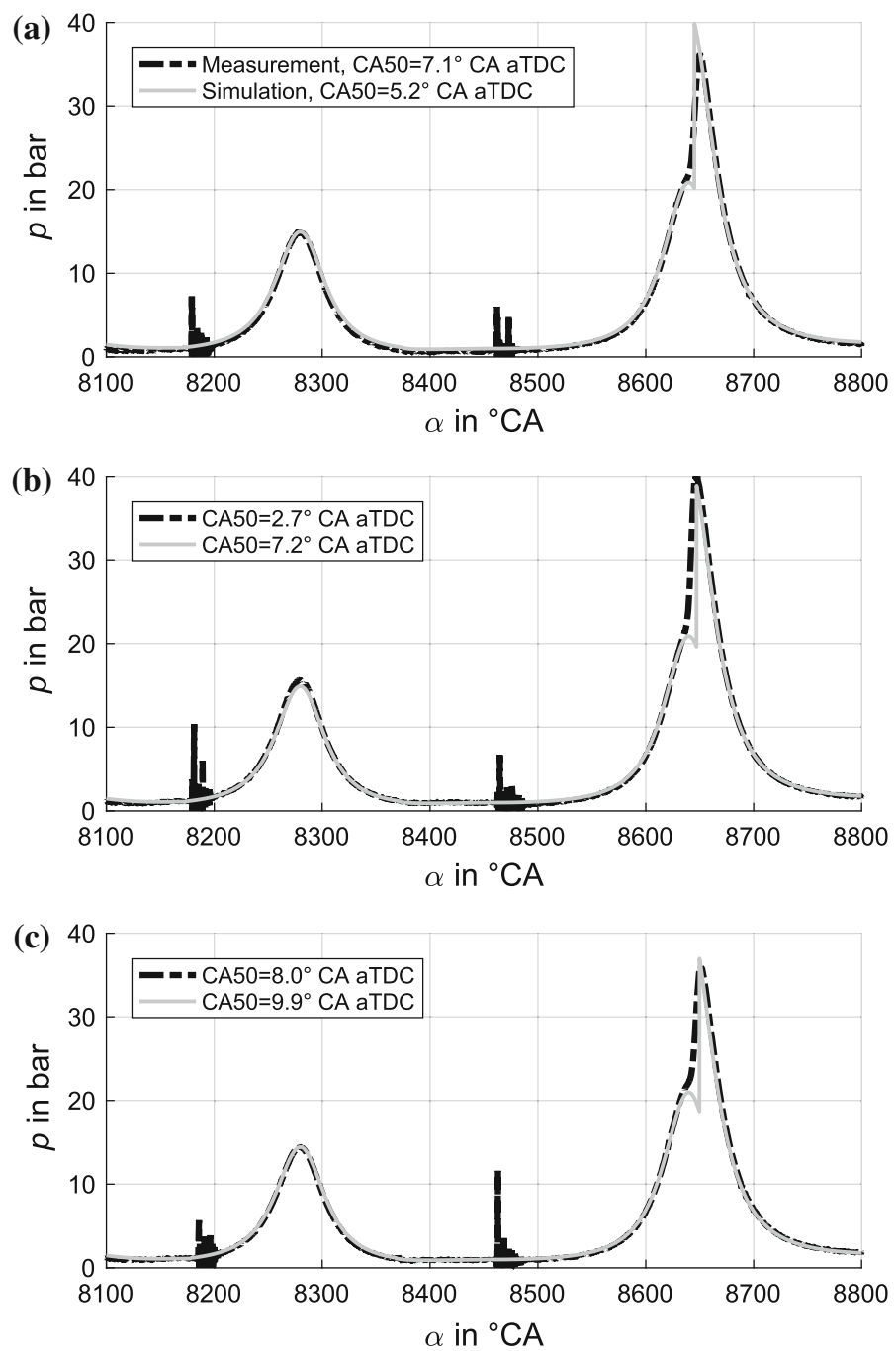

Fig. 4 Simulated versus measured pressure trace. a Measurement point 1. b Measurement point 2. c Measurement point 3

between an open loop simulation of the presented model and another experimental GCAI setup. In the simulation, $\mathrm{NVO}$ is further decreased to $170^{\circ} \mathrm{CA}$ and a disturbance in form of white noise is added to the initial condition of the in-cylinder temperature at inlet valve closing. Again, $\alpha_{s o i}$ is referenced to IVO and the remaining control inputs stay as before. The simulation outcome of this setup shows a clear correspondence to the experimental data. In general, the characteristic change in the dynamic behaviour can thus be reproduced by the derived model. Further research is 

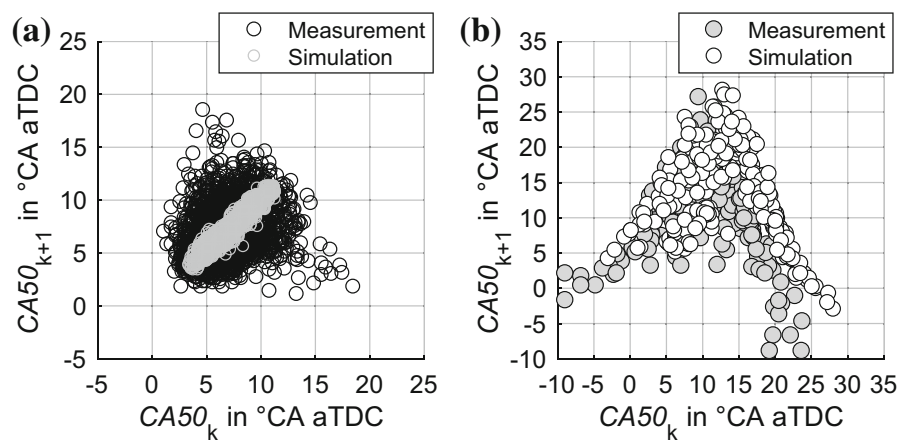

Fig.5 Return map of center of combustion CA50. a Simulated against measured data with measured input. b Qualitative comparison between simulation and measurement for small NVO and late combustion

necessary to adapt the moment of transition to the same point as for the experimental data in Fig. 5 a.

The averaged computation time for simulation of one cycle on an Intel i7 processor using Simulink's ode3 (Bogacki-Shampine) is $250 \mathrm{~ms}$. Consequently, admissible simplifications must be found for the model to be suitable for control synthesis. In case of model predictive control, which has shown to have favourable properties for the present control problem [11], the model is used in context of optimization and consequently needs to be as simple as possible. Furthermore, the prediction of the ignition delay has to be improved, as there is a discrepancy with regard to the values determined by heat release analysis. As the overall pressure trace shows good correspondence, however, the continuous-cycle model allows using other metrics than CA50 for controlling the process. If the onset of the cyclic variations seen in Fig. $5 \mathrm{~b}$ is to be avoided by the controller, a proper tuning of the parameters needs to be conducted, so the predicted change in system dynamics occurs at the same conditions as at the test bed.

\section{Conclusions}

The paper presents a modeling approach for GCAI with the purpose of multi-scale control synthesis. In this particular case, it is required to model the process timeresolved, instead of the cycle-to-cycle resolution of state-of-the-art mean-value models. The paper provides a physically motivated, control-oriented GCAI model which is continuous in time. A comparison between the simulated model output and the continuous pressure traces of a selected set of measurement data verifies the general assumptions made. The in-cycle resolution of the combustion cycle allows for new control approaches such as in-cycle control and therefore motivates further research. In particular, the ignition delay model must be improved as the prediction of CA50 is 
not accurate. In addition, further work needs to be conducted concerning the reduction of complexity. Despite the comparably low order, the simulation time of $250 \mathrm{~ms}$ for an engine cycle is excessively high. Finally, the model parameters need to be identified and the covered operating range of model assessed.

Acknowledgements The research was performed as part of the Research Unit (Forschergruppe) FOR2401 "Optimization based Multiscale Control for Low Temperature Combustion Engines", which is funded by the German Research Association (Deutsche Forschungsgemeinschaft, DFG). The support is gratefully acknowledged.

\section{References}

1. Lehrheuer, B., Pischinger, S., Wick, M., Andert, J., Berneck, D., Ritter, D., Albin, T., Thewes, M.: A study on in-cycle combustion control for gasoline controlled autoignition. In: SAE Technical Paper 2016-01-0754 (2016)

2. Yao, M., Zheng, Z., Liu, H.: Progress and recent trends in homogeneous charge compression ignition (HCCI) engines. Progress Energy Combust. Sci. 35 (2009)

3. Ebrahimi, K., Koch, C.: Model predictive control for combustion timing and load control in HCCI engines. In: SAE Technical Paper 2015-01-0822 (2015)

4. Jade, S., Hellstöm, E., Larimore, J., Stefanopoulou, A.G., Jiang, L.: Reference governor for load control in a multicylinder recompression HCCI engine. IEEE Trans. Control Systems Technol. 22(4) (2014)

5. Shaver, G.M., Gerdes, J.C., Jain, P., Caton, P.A., Edwards, C.F.: Modeling for control of HCCI engines. In: Proceedings of the American Control Conference (2003)

6. Jade, S., Larimore, J., Hellstöm, E., Stefanopoulou, A.G.: Controlled load and speed transitions in a multicylinder recompression HCCI engine. IEEE Trans. Control Syst. Technol. 23(3) (2015)

7. Jade, S., Larimore, J., Hellstöm, E., Jiang, L., Stefanopoulou, A. G.: Enabling large load transitions on multicylinder recompression HCCI engines using fuel governors. In: American Control Conference (ACC), Washington, USA (2013)

8. Hohenberg, G.F.: Advanced approaches for heat transfer calculations. In: 1979 SAE International Off-Highway and Powerplant Congress and Exposition (1979)

9. Shahbakhti, M., Koch, C.R.: Characterizing the cyclic variability of ignition timing in a homogeneous charge compression ignition engine fuelled with n-heptane/iso-octane blend fuels. J. Int. Engine Res. 9 (2008)

10. Ritter, D., Andert, J., Abel, D., Albin, T.: Model-based control of gasoline-controlled autoignition. Int. J. Engine Res. 19 (2018)

11. Albin, A., Zweigel, R., Hesseler, F.: A 2-stage MPC approach for the cycle-to-cycle dynamics of GCAI (Gasoline Controlled Auto Ignition). In: 7th IFAC Symposium on Advances in Automotive Control (2013) 